\title{
Prácticas docentes y cultura inclusiva para colectivos vulnerables de primarias en Sonora, México
}

\author{
Reyna de los Ángeles Campa-Álvarez, Ph.D. \\ Universidad de Sonora, México* \\ Blanca Aurelia Valenzuela, Ph.D. \\ Universidad de Sonora, México** \\ Manuela Guillén-Lúgigo, Ph.D.

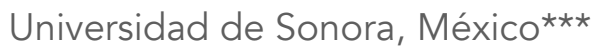

reyna.campa@unison.mx

\section{Resumen (analítico)}

El objetivo de la investigación fue analizar las prácticas y cultura inclusiva de docentes en educación primaria para la atención de colectivos vulnerables en Hermosillo, Sonora, México. La metodología fue de corte cuantitativa no experimental y se aplicó el cuestionario Índice de inclusión a 124 profesores y profesoras. Los resultados obtenidos reportan que los tipos de vulnerabilidad más comunes en el aula corresponden a una situación económica baja, familia disfuncional y discapacidad. Se muestra una relación significativa bidireccional entre práctica y cultura inclusiva (.741) por lo que se requiere crear una comunidad colaborativa, implementar metodologías inclusivas y recursos de apoyo. El modelo estructural predice un $36 \%$ la educación inclusiva de los colectivos vulnerables.

\section{Palabras clave}

Inclusión, vulnerabilidad, infancia, prácticas, cultura.

\section{Tesauro}

Tesauro de Ciencias Sociales de la Unesco.

\section{Para citar este artículo}

Campa-Álvarez, R de los Á., Valenzuela, B. A., \& Guillén-Lúgigo, M. (2020). Prácticas docentes y cultura inclusiva para colectivos vulnerables de Primarias en Sonora, México. Revista Latinoamericana de Ciencias Sociales, Niñez y Juventud, 18(2), 1-17 http://dx.doi.org/10.11600/1692715x.18211

\section{Historial}

Recibido: 02.10.2019

Aceptado: 10.02 .2020

Publicado: 30.05 .2020

\section{Información artículo}

Artículo del proyecto en curso «Educación inclusiva para la atención de estudiantes a nivel básico en condición de vulnerabilidad Social en el estado de Sonora, México» (Folio: Unison-PTC-297), financiado por Programa para el Desarrollo Profesional Docente, Tipo Superior del Gobierno de México. Se reportan resultados del pilotaje realizado en el año 2019. Área: Ciencias Sociales; subárea: Psicología. 


\section{Teaching practices and inclusive culture for vulnerable primary school groups in Sonora, Mexico}

\section{Abstract (analytical)}

The objective of this research was to analyze the practices and inclusive culture of primary school teachers who work with children from vulnerable population groups in Hermosillo, Sonora, Mexico. The methodology used was a non-experimental quantitative study. The Inclusion Index questionnaire was completed by 124 teachers. The results show that the most common types of vulnerability in the classroom correspond to poor economic situations, dysfunctional families and disabilities. There is a significant bidirectional relationship between teaching practices and inclusive culture (.741), which means that it is necessary to create a collaborative community, implement inclusive methodologies and support resources. The structural model predicts $36 \%$ inclusive education for students from vulnerable population groups.

Keywords

Inclusion, vulnerability, childhood, practices, culture.

\section{Práticas docentes e cultura inclusiva para grupos vulneráveis de primários em Sonora, México}

\section{Resumo (analítico)}

O objetivo da pesquisa foi analisar as práticas e cultura inclusiva de professores do ensino fundamental para a atenção de grupos vulneráveis em Hermosillo, Sonora, México. A metodologia foi de corte quantitativo não experimental e o questionário do Índice de Inclusão foi aplicado a 124 professores. Os resultados obtidos relatam que os tipos mais comuns de vulnerabilidade em sala de aula correspondem a uma baixa situação econômica, família disfuncional e incapacidade. Há uma relação bidirecional significativa entre a prática e a cultura inclusiva (.741); portanto, é necessário criar uma comunidade colaborativa, implementar metodologias inclusivas e recursos de apoio. $\bigcirc$ modelo estrutural prevê 36\% de educação inclusiva para grupos vulneráveis.

\section{Palavras-chave}

Incluso, vulnerabilidade, infância, práticas, cultura.

\section{Información autoras}

[*] Psicóloga, Maestra y Doctora en Ciencias Sociales por la Universidad de Sonora (México). Profesorainvestigadora de tiempo completo del Departamento de Psicología y Ciencias de la Comunicación, Universidad de Sonora (México). (iD) 0000-0003-3780-9623. Índice H5: 3. Correo electrónico: reyna.campa@unison.mx

[**] Psicóloga y Doctora en Filosofía y Ciencias de la Educación por Universidad Nacional de Educación a Distancia (España). Profesora-investigadora de tiempo completo del Departamento de Psicología y Ciencias de la Comunicación, Universidad de Sonora (México). (iD) 0000-0003-0960-9499. Índice H5: 4. Correo electrónico: blanca.valenzuela@unison.mx

[***] Doctora en Ciencias Políticas y Sociología por la Universidad Nacional de Educación a Distancia (España). Profesora-investigadora de tiempo completo del Departamento de Trabajo Social, Universidad de Sonora (México). iD 0000-0002-7801-5996. Índice H5: 5. Correo electrónico: manuela.guillen@unison.mx 


\section{Introducción}

ـ a inclusión es un nuevo modelo educativo que pretende brindar atención para Es preciso indicar que la educación inclusiva atiende a personas en condición de pobreza, género, interculturalidad, etnia y discapacidad, entre otros (Barrero, 2016; Echeita \& Ainscow, 2011; Guillén \& Valenzuela, 2015), representando colectivos sociales con menores oportunidades de acceso a la educación básica, la cual es fundamental cursar para contribuir en el desarrollo intelectual y social del individuo (Blanco, 2015). Al referirse la expresión vulnerabilidad social se puede aludir a un proceso de exclusión acentuado que repercute de forma amplia en el rezago social, educativo y bienestar de grupos poblacionales que no alcanzan los beneficios de inversión social (Ordóñez, 2018).

Cifras estadísticas en México revelan un panorama urgente de atención e intervención en estos grupos de estudiantes: el Consejo Nacional de Evaluación de la Política de Desarrollo Social (2016) indica que en el estado de Sonora, México, el porcentaje de población que se encuentra en situación de pobreza es de $32.8 \%$ (875 ooo) y en pobreza extrema es del 5.3\% (142 ooo). Por su parte, los datos de la Encuesta de Salud y Nutrición (2016) indican que en Sonora el 30.9\% de las niñas, niños y adolescentes habitan en hogares donde existe carencia por acceso a la alimentación; dicha cifra muestra la mayor vulnerabilidad que enfrenta la población infantil en materia de alimentación y nutrición; lo que tiene consecuencias en el desarrollo del individuo.

Por otra parte, el Instituto Nacional de Estadística y Geografía (2015) indica que cerca de 10704 niñas y niños de o a 14 años (equivalentes al $1.4 \%$ de la población infantil) tienen dificultades para realizar actividades cotidianas, dado que presentan algún tipo de discapacidad. Datos de la Secretaría de Educación Pública (2018) muestran un registro de estudiantes inscritos en el nivel de educación básica de 568 332; 98752 pertenecientes a preescolar (17.37\%), 317692 a primaria $(55.89 \%)$ y 151888 a secundaria $(26.72 \%)$ 
durante el ciclo 2016-2017. Se puede apreciar una diferencia de cifras entre primaria y secundaria, donde se detecta el abandono escolar entre un nivel y otro casi del 50\%.

Ante tal panorama, se evidencian indicadores de vulnerabilidad y riesgo de exclusión socioeducativa. La falta de acceso a la institucionalización, participación o reducción de los espacios sociales genera efectos de desigualdad para una vida digna y plena; lo que sitúa a los colectivos vulnerables en una lucha constante para legitimar sus derechos humanos fundamentales (Fjeld \& Quintana, 2019). Frente a esta perspectiva, surge la importancia de que los centros educativos sean justos y equitativos, siendo elemental, según Subirats (2013), que en este proceso de inclusión se reconstruya la condición de los actores sociales, la cual es una acción compartida por la comunidad y sociedad en general.

En México los retos de la educación inclusiva tienen relación con la construcción de políticas, prácticas y culturas inclusivas que permitan a la diversidad de estudiantes alcanzar los aprendizajes básicos en este nivel obligatorio (Marshesi et al., 2014; Montiel \& Arias, 2017; Valenzuela et al., 2014). Según la Secretaría de Educación Pública (2017), se entiende educación inclusiva como:

La valoración de la diversidad, adaptando el sistema para responder de manera adecuada a las necesidades de todos y cada uno de los estudiantes. En ese sentido, se pretende asegurar la equidad y la calidad en la educación, considerando a todos los alumnos sin importar sus características, necesidades, intereses, capacidades, habilidades y estilos de aprendizaje. (p. 21)

Por su parte, Booth y Ainscow (2011) señalan que la educación inclusiva hace referencia a un proceso que pretende reducir las barreras del aprendizaje y la participación de todos los estudiantes. Dicho proceso consta de tres dimensiones: 1) cultura; 2) política; y, 3) práctica. La dimensión cultural se refiere a la comunidad educativa con valores y creencias compartidas; en la segunda dimensión la inclusión es el impulsor del centro educativo y delimita las modalidades de apoyo para dar frente a la diversidad; y en la tercera, las prácticas promueven las actividades de participación plena y efectiva acorde a la cultura y capacidades del alumno.

Por lo anterior, es necesario dar un valor esencial a la educación inclusiva como una acción de intervención psicopedagógica y protectora de riesgos sociales para facilitar los aprendizajes en estudiantes con desventaja (Casanova, 2017). De acuerdo con esta propuesta, en una investigación realizada por Campa y Contreras (2018), se señalan como ejes medulares en el proceso de inclusión las actitudes, prácticas y culturas inclusivas de docentes en 
educación básica, enmarcado la igualdad de derecho y la promoción de valores en el aula. Por su parte, Barriga et al. (2019) atribuyen las expectativas, la formación y vocación docente como elementos para desarrollar el potencial en todos los estudiantes, independientemente de su diversidad humana.

Un estudio realizado por Serrano et al. (2019) plantea que los centros escolares deben ser vistos como espacios inclusivos en los que se reconozca a cada persona como un ser único y que permitan atender la variedad estudiantil a partir de fundamentar las prácticas y participación entre los actores escolares. Para la efectividad de las prácticas inclusivas es necesario modificar la organización escolar, a través de un clima cálido, cómodo y propicio para el aprendizaje, en el que los estudiantes tengan la confianza de expresar sus inquietudes y necesidades. Para ello la labor docente es clave, por la constante interacción didáctica que establece con los estudiantes y el empleo de estrategias en el aula (Cara et al. 2015; Echeita, 2013; Euan \& Echeverría, 2016).

Es importante considerar que la comunidad educativa es responsable del proceso de formación de los individuos, así como del ambiente en el que estudian los niños y niñas. También se debe tener en cuenta que a través de los medios escolares se propician los apoyos requeridos para el aprendizaje. Para dicha formación es imprescindible la construcción de culturas de inclusivas con aprecio a la diversidad humana, con significados y prácticas institucionales dirigidas a que todos los estudiantes sientan la seguridad y convicción de alcanzar sus metas (Romero et al., 2018; López, 2018). Se entiende la expresión cultura inclusiva como el conjunto de normativas, símbolos, creencias y valores compartidos por la comunidad escolar (estudiantes, directores, docentes, administrativos y padres de familia) para configurar una comunidad segura, abierta, colaboradora y estimulante en la que todos sean valorados y aceptados (Booth \& Ainscow, 2011; Mateus et al., 2017).

Lo anterior resalta la necesidad de implementar acciones significativas que incidan de manera individual y grupal en los aprendizajes de niños y niñas. González y Londoño (2019) señalan que se debe tener en cuenta el contexto sociocultural donde se desenvuelven los y las estudiantes para contribuir positivamente en el fortalecimiento académico, así como la participación e interés de estudiantes y docentes involucrados en el proceso inclusivo.

Mencionado lo anterior, la investigación se centra en el estudio del nivel educativo de primaria al ser una de las etapas más significativas del ser humano. En este periodo no solamente se adquieren los aprendizajes y conocimientos intelectuales básicos, sino que también el individuo adquiere normas de convivencia social, desarrolla su autoestima, 
habilidades para la integridad humana y la creación de un proyecto de vida, principalmente en aquellos estudiantes que enfrentan una situación vulnerable.

En ese sentido la labor docente se considera un pilar fundamental para el desarrollo de una educación inclusiva. El problema radica cuando se cuestiona lo siguiente: ¿̨los y las docentes de educación primaria realizan prácticas pertinentes para una cultura inclusiva que impacte positivamente en los colectivos vulnerables? Para dar respuesta a dicha cuestión se tiene como objetivo general analizar las prácticas y cultura inclusiva efectuada por docentes de educación primaria pública en el estado de Sonora, México, para la atención de estudiantes que forman parte de colectivos vulnerables, a fin de detectar los principales indicadores de inclusión y las áreas que requieren mayor desarrollo para el fomento de una educación inclusiva.

Para cumplir con el objetivo se utilizó una metodología cuantitativa. Se aplicaron cuestionarios a profesores y profesoras de escuelas primarias públicas en Hermosillo, Sonora, México. Se acudió a zonas geográficas vulnerables y a reuniones del Consejo Técnico del Profesional Docente de Educación Básica para complementar la fase de pilotaje. Con los resultados reportados se ponen en relieve los indicadores de prácticas docentes y cultura inclusiva que requieren de un entrenamiento para brindar una respuesta educativa acorde a las necesidades de los colectivos vulnerables en primarias públicas.

\section{Método}

La presente investigación tiene un enfoque cuantitativo, el cual emplea la medición numérica y el análisis estadístico de datos, lo que permite establecer patrones de comportamiento en una población o grupo de individuos (Hernández et al., 2010). El tipo de estudio es descriptivo-correlacional (Monje, 2011). En este caso se tienen como variables de estudio las prácticas docentes y la cultura inclusiva. El diseño de investigación fue no experimental debido a que las variables no fueron manipuladas; se basó en la observación del contexto natural y los participantes no fueron expuestos a estímulos.

\section{Muestra}

Para la conformación de la fase de pilotaje reportada en la investigación se realizó un muestreo no probabilístico intencional, el cual se conformó por 124 profesores y profesoras de educación primaria pública del municipio de Hermosillo. Se acudió a escuelas 
ubicadas en zonas geográficas vulnerables con altos índices de pobreza y marginación. Los sectores seleccionados fueron: Las Amapolas, La Costa, San Luis, La Victoria, Palo verde y Café Combate. Así mismo, participaron profesores que acudieron a las reuniones del Consejo Técnico del Profesional Docente de Educación Primaria. Del total de participantes, un $58 \%(\mathrm{~N}=72)$ son mujeres y un $42 \%(\mathrm{~N}=52)$ son hombres. Referente al nivel educativo de los y las docentes, un $68 \%$ cuenta con estudios de licenciatura, $12 \%$ maestría, $8 \%$ especialidad y un $12 \%$ diplomado. Los años de antigüedad docente oscilan de 1 a 35 años, con una media de 10-15 años (D.E.= 2.123). Para efectos del estudio los criterios de selección de los participantes fueron los siguientes: 1) escuelas primarias públicas; 2) ubicadas en zonas geográficas vulnerables; 3) se consideraron todos los grados educativos de primero a sexto.

\section{Instrumentos}

Se aplicó el cuestionario Índice de inclusión (Booth \& Ainscow, 2000) que mide tres dimensiones: a) cultura inclusiva; b) políticas inclusivas; y, c) prácticas inclusivas. Consta de 45 reactivos con opciones de respuesta tipo Likert. Para los propósitos de la investigación se emplearon las dimensiones a y c. El impacto internacional del índice ha generado que se desarrolle en diversos países, en tanto que ha sido propuesto por la Unesco como una medida para favorecer la inclusión. Por el contexto del estudio se realizó una adaptación a las características idiosincráticas de México y a la región noroeste del país, a través de la revisión de expertos, prueba piloto y análisis de confiabilidad alfa de Cronbach, obteniendo un total de .82 , lo que demuestra una consistencia interna altamente aceptable.

\section{Procedimiento y análisis de datos}

Para la aplicación de los cuestionarios se acudió primeramente a los respectivos directivos para obtener la autorización de realizar el estudio. Una vez obtenida su autorización, se prosiguió a reunir a los profesores para tener su consentimiento para participar. La aplicación fue a lápiz y papel, con una duración aproximada entre 15 a 20 minutos; se les indicó que respondieran con la mayor veracidad y se garantizó el anonimato. Una vez concluida la recolección de datos, se utilizó el programa estadístico SPSS ${ }^{\text {тм }}$ (v. 23) a fin de realizar un análisis de frabilidad, descriptivo y correlacional. También se utilizó el programa $\mathrm{EQS}^{\mathrm{TM}}$ (v. 6.1) para realizar el modelo estructural. Esta es una técnica estadística multivariada para representar interrelaciones entre variables y analizar la influencia directa e indirecta entre las mismas (Bentler, 2006). Por último, para determinar la per- 
tinencia del modelo propuesto, se utilizaron los indicadores de bondad de ajuste chi cuadrado, así como los indicadores prácticos Bentler-Bonett de ajuste normado (BBNFI) y Bentler-Bonettt de ajuste no normado (BBNNFI).

\section{Resultados}

Los resultados indican que los y las docentes han atendido en mayor porcentaje los siguientes tipos de vulnerabilidad estudiantil: situación de pobreza (62\%), discapacidad visual (45\%), problemas de aprendizaje (38\%). Por otra parte, en menor porcentaje se ha atendido a estudiantes con discapacidad motora (15\%), discapacidad auditiva (8\%), autismo ( $5 \%$ ) y migrantes ( $5 \%$ ) (figura 1$)$.

\section{Figura 1}

\section{Tipos de vulnerabilidad atendida por el profesorado de primaria pública}

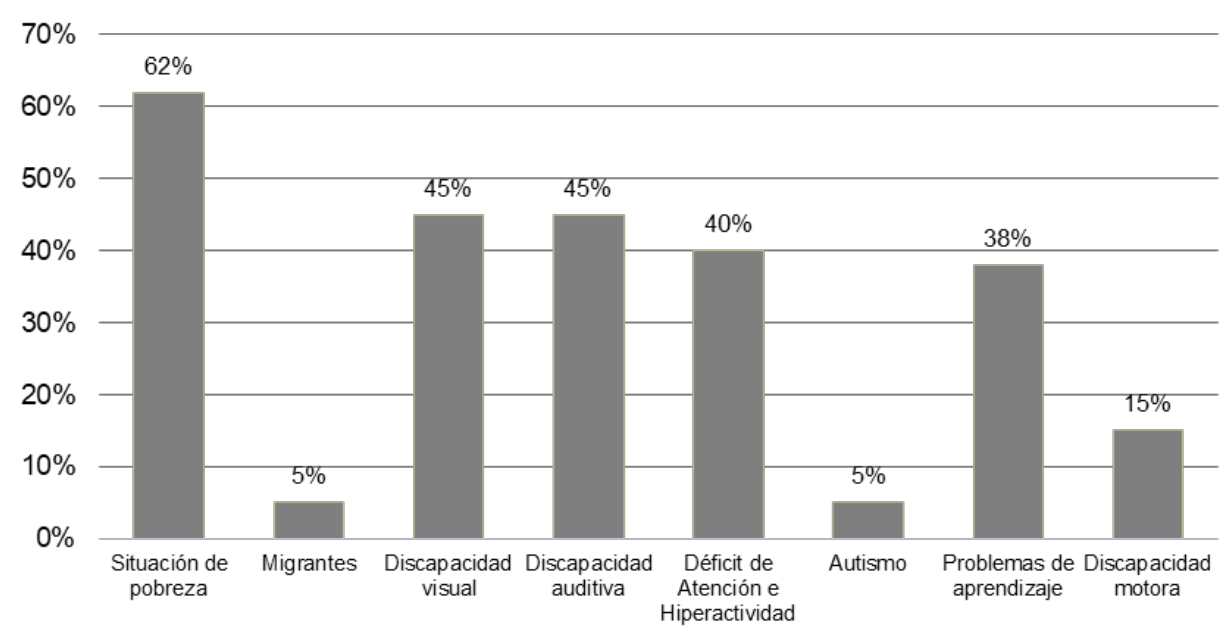

Para la validez del instrumento se obtuvo el alfa de Cronbach de las escalas de práctica y cultura inclusiva que integran el índice de inclusión; los resultados se muestran en la tabla 1. Como se puede observar, el alfa total del instrumento fue de .82 , lo que indica una alta fiabilidad. De igual forma, se muestran los datos descriptivos de las variables de dichas escalas. Respecto a las prácticas inclusivas, las medias altas se obtuvieron en planificaciones y adaptaciones curriculares $(\bar{X}=3.45$; D.E. $=.727)$, participación activa de los estudiantes en el aprendizaje $(\bar{X}=3.42$ D.E. $=.605)$ y evaluaciones adaptadas a las necesi$\mathrm{dad}(\bar{X}=3.24$; D.E. $=.729)$; mientras que las medias bajas fueron en recursos de apoyo en el proceso de inclusión $\left(\bar{X}_{=1.90}\right.$ D.E. $\left.=.984\right)$ y metodología inclusiva $(\bar{X}=2.71 ;$ D.E. $=878)$. 
Los resultados descriptivos de cultura inclusiva arrojan como medias más altas el aprecio a la diversidad $(\bar{X}=3.97$, D.E. $=.658)$, seguido de la promoción de valores inclusivos $(\bar{X}=3.90$, D.E. $=.773)$ y, por último, disminución de prácticas discriminatorias $(\bar{X}=3.81$, D.E. $=.744)$. Por otra parte, las medias más bajas se encontraron en la creación de una comunidad colaborativa $(\bar{X}=2.37$, D.E. $=.625)$, así como en la falta de coordinación y apoyo $(\bar{X}=1.97$, D.E. $=.685)$.

\section{Tabla 1}

\section{Datos descriptivos y fiabilidad de las escalas del instrumento}

\begin{tabular}{lcccccc}
\hline Escalas/variables & N & Min & Max & Media & D.E. & Alfa \\
\hline \multicolumn{1}{c}{\begin{tabular}{l} 
Índice de inclusión \\
\multicolumn{1}{c}{ Prácticas inclusivas }
\end{tabular}} & & & & & & $\mathbf{8 2}$ \\
& & & & & .87 \\
Planificaciones y adaptaciones curriculares & 124 & 1 & 4 & 3.45 & .727 \\
Metodología inclusiva & 124 & 1 & 4 & 2.71 & .878 \\
$\begin{array}{l}\text { Participación activa de los estudiantes en el } \\
\text { aprendizaje }\end{array}$ & 124 & 1 & 4 & 3.42 & .605 \\
Recursos de apoyo para la inclusión & 124 & 1 & 4 & 1.90 & .749 \\
Evaluaciones adaptadas a las necesidades & 124 & 1 & 4 & 3.42 & .605 \\
Cultura inclusiva & & & & & \\
Comunidad colaborativa & 124 & 1 & 4 & 2.37 & .625 \\
Valores inclusivos & 124 & 1 & 4 & 3.90 & .773 \\
Coordinación y apoyo & 124 & 1 & 4 & 1.97 & .625 \\
Aprecio a la diversidad & 124 & 1 & 4 & 3.97 & .773 \\
Disminución de prácticas discriminatorias & 124 & 1 & 4 & 3.81 & .744 \\
\hline
\end{tabular}

$\mathrm{Al}$ analizar los indicadores de la variable prácticas inclusivas, la figura 2 muestra los porcentajes en relación con los recursos implementados para el apoyo del proceso de inclusión, siendo bajo en los recursos por la comunidad (11\%) y por parte del personal en apoyo al aprendizaje y participación del estudiante (27\%); por otra parte, se obtuvo un mayor porcentaje en recursos empleados para la convivencia escolar (46\%) y la diversidad es utilizada como recurso para la enseñanza-aprendizaje (39\%). Así mismo, se obtu- 
vo un mayor porcentaje en recursos empleados para el apoyo de la convivencia escolar (36\%) y la diversidad es utilizada como recurso para la enseñanza-aprendizaje (26\%).

\section{Figura 2}

\section{Porcentaje de recursos implementados para el apoyo del proceso de inclusión}

El personal genera recursos para apoyar el aprendizaje y participación La diversidad se utiliza como un recurso de enseñanza-aprendizaje Recursos de apoyo por la comunidad Recursos de apoyo para la convivencia

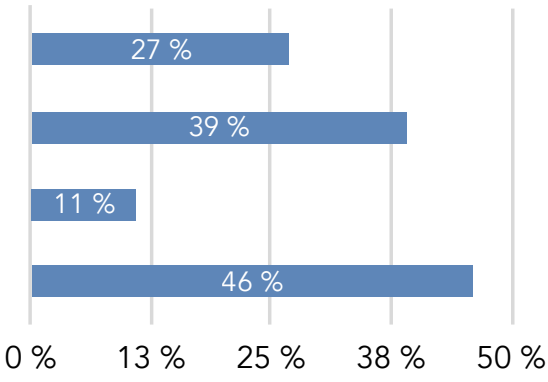

En lo que respecta a la metodología inclusiva como parte de las prácticas inclusivas, lo relacionado a las funciones que realiza el profesorado en el aula puede indicar medias bajas en las planificaciones, el desarrollo de la clase responde a la diversidad del alumnado $(\bar{X}=2.28)$ y las clases son accesibles para todos $(\bar{X}=2.14)$; representando indicadores que requieren potencializarse para brindar una atención acorde a la vulnerabilidad estudiantil. Por otra parte, los indicadores con medias altas puntuaron en que la disciplina en el aula se basa en el respeto mutuo $(\bar{X}=3.79)$, los estudiantes aprenden de manera cooperativa $(\bar{X}=3.73)$, la evaluación estimula los logros $(\bar{X}=3.32)$ y se implica activamente el alumno en el aprendizaje $(\bar{X}=3.45)$ (figura 3$)$.

\section{Figura 3}

Medias de metodología inclusiva del profesorado de primaria en atención a grupos vulnerables

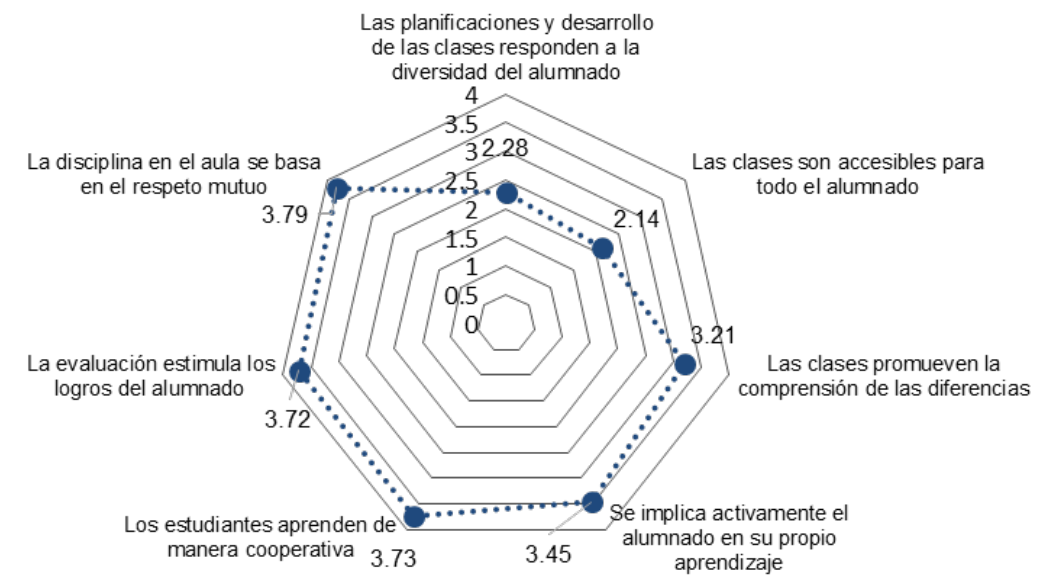


Por otra parte, en la figura 4 se muestran los indicadores de comunidad colaborativa dentro de la escala cultura inclusiva, presentándose una media baja en los miembros del personal que colaboran entre sí $(\bar{X}=2.24)$, una tendencia favorable en las relaciones establecidas entre estudiantes y profesores al indicar que se tratan con respeto $(\bar{X}=3.65)$ y entre iguales niños y niñas que se ayudan entre sí, $(\bar{X}=3.56)$ motivo por el cual se sienten cómodos en la escuela $(\bar{X}=3.15)$. Así mismo, se detecta en los indicadores de coordinación y apoyo como parte de la cultura inclusiva medias bajas, principalmente en la escasa o nula participación por parte de las instituciones de la localidad en actividades del centro educativo $(\bar{X}=1.24)$, seguido de la poca relación establecida por los familiares de los estudiantes, principalmente de aquellos en una situación vulnerable $(\bar{X}=1.84)$; también se puede señalar la necesidad de trabajar en conjunto tanto del personal como de los miembros del consejo escolar $(\bar{X}=2.34)$.

\section{Figura 4}

\section{Medias de comunidad colaborativa, coordinación y apoyo en atención a grupos vulnerables}

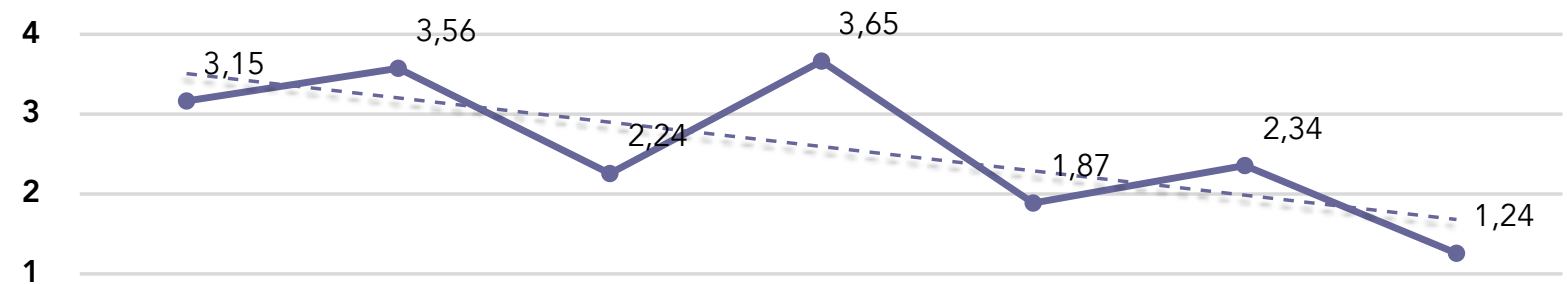

0

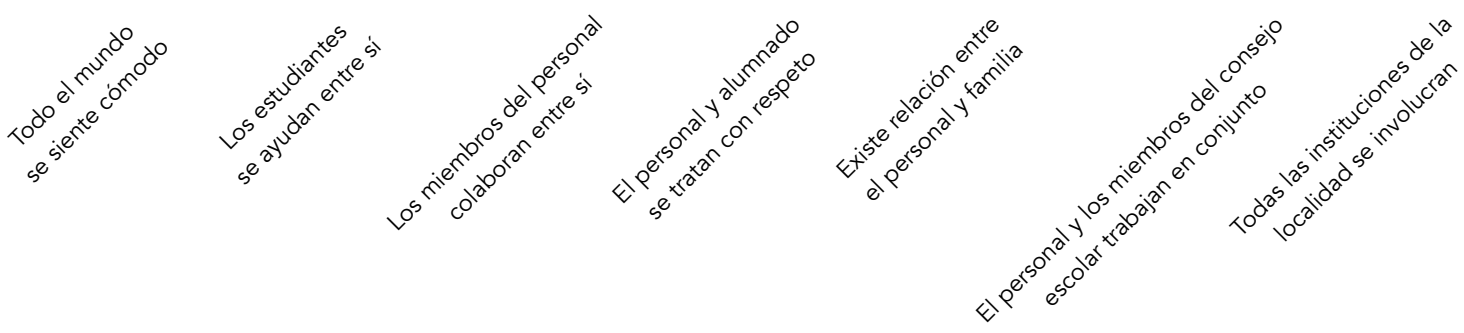

Los resultados de las correlaciones de Pearson (tabla 2) muestran un nivel de significancia $\mathrm{o}, \mathrm{O1}$ (bilateral), por lo que se pude decir que existe una relación altamente significativa entre prácticas inclusivas y cultura inclusiva $\left(.741^{* *}\right)$.

En la figura 5 se muestran los resultados del modelo estructural. Se identifica una relación bidireccional entre el factor de práctica y la cultura inclusiva, con una covarianza de .69. Se obtuvo validez convergente en los constructos de cada factor con pesos estructurales mayores de .15 , los cuales indican significancia. 


\section{Tabla 2}

\section{Correlaciones entre las variables de estudio}

\begin{tabular}{lcc} 
& $\begin{array}{c}\text { Prácticas } \\
\text { inclusivas }\end{array}$ & $\begin{array}{c}\text { Cultura } \\
\text { inclusiva }\end{array}$ \\
\hline Prácticas inclusivas & 1 & - \\
Cultura inclusiva & $.741^{\star \star}$ & 1 \\
\hline
\end{tabular}

Nota. $* *$ la correlación es significativa al nivel o,o1 (bilateral).

Los indicadores de bondad de ajuste se consideran aceptables, ya que los valores obtenidos fueron los siguientes: índice de ajuste normado de Bentler-Bonett $(\mathrm{BBNFI})=.93$, índice de ajuste no normado de Bentler-Bonett $(\mathrm{BBNNFI})=.94 \mathrm{y}$ el índice comparativo de ajuste de Bentler $(\mathrm{CFI})=.94$. Estos valores alcanzan el puntaje mínimo considerado aceptable en los indicadores prácticos para considerar un modelo con buena bondad de ajuste a partir de .90 .

\section{Figura 5}

\section{Modelo estructural de las variables de estudio}

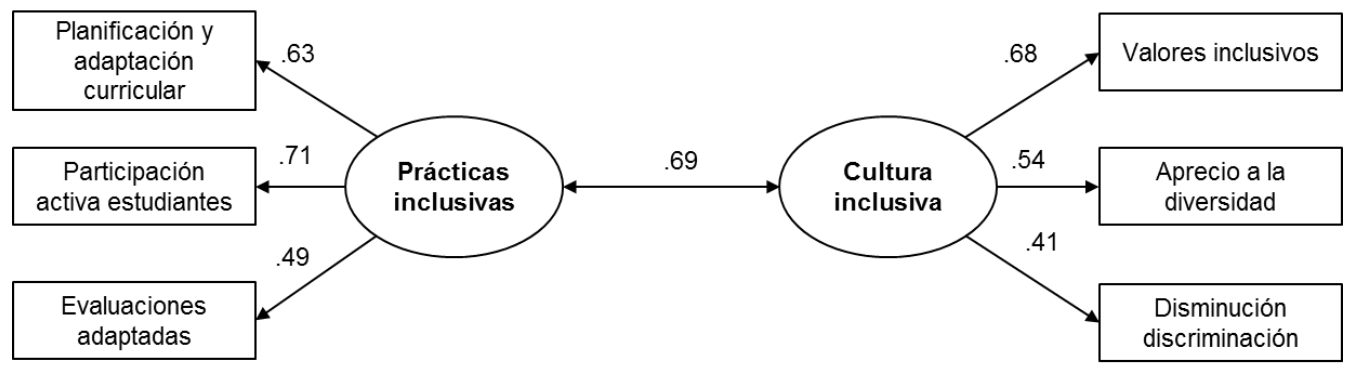

$X^{2}=37.447$ (12 gl. $) \quad p=.001 \quad \mathrm{BBNFI}=.93 \quad \mathrm{BBNNFI}=.94 \quad \mathrm{CFI}=.94 \quad \mathrm{RMSEA}=.059 \quad R^{2}=.36$

El valor del chi cuadrado fue de $\mathrm{X}^{2}=37.44$ (12 gl). $\mathrm{p}=0.001$. La probabilidad no fue significativa, lo que indica que no existen diferencias entre el modelo teórico y el modelo saturado por las relaciones entre variables. Por último, se obtuvo un valor de .o59 en RMSEA (residuo de cuadrados mínimos), lo cual está dentro de los parámetros considerados aceptables .o8; mientras que la $\mathrm{R}^{2}$ fue .36, lo que significa que el modelo en su conjunto explica el $36 \%$ de la varianza práctica y cultura inclusiva. 


\section{Discusión}

Con base en los resultados obtenidos se cumplió con el objetivo general y pregunta de investigación planteada al evidenciar las prácticas inclusivas efectuadas por los y las docentes, las cuales son: adaptaciones curriculares y evaluaciones adaptadas a la vulnerabilidad de los estudiantes, destacando la participación activa de los mismos. Sin embargo, se encontró una necesidad y carencia en recursos de apoyo para la inclusión, así como la falta de empleo de metodologías inclusivas, estableciéndose como factores que interfieren en una educación inclusiva de calidad. Se detectó que el tipo de vulnerabilidad más común que se atiende es de orden social en relación a una situación económica baja - en algunos casos de pobreza extrema-, lo cual deriva en problemas de aprendizaje y, aunado a ello, el tener una familia disfuncional afecta el desempeño de los estudiantes en el aula. Por otro lado, también se detectó en los niños y niñas inscritos en las primarias participantes algún tipo de discapacidad (visual, motora, intelectual o auditiva) y déficit de atención e hiperactividad.

Ocampo (2019) indica que cursar una educación inclusiva a nivel básico favorece el desarrollo de niños y niñas, reduciendo en etapas tempranas las desigualdades sociales, económicas y barreras educativas, además de la incidencia que tienen las prácticas inclusivas en las trayectorias escolares al disminuir los índices de deserción y rezago educativo. Se impacta en mayor medida en el aprendizaje cuando se ofrecen contenidos académicos adaptados, se propician interacciones colaborativas entre los miembros, se cuenta con recursos materiales que facilitan la inclusión y se reciben constantes capacitaciones en atención a los grupos más vulnerables (Jiménez et al., 2017).

En lo que respecta a la cultura inclusiva, se pueden señalar indicadores de aprecio a la diversidad, la promoción de valores en el centro escolar y la disminución de prácticas discriminatorias. Sin embargo, aún se requiere crear una comunidad colaborativa entre los miembros de la escuela y la sociedad, así como coordinar las formas de apoyo para la inclusión de manera que sean efectivas para todos los actores involucrados en el proceso. Leithwood (2009) plantea una planificación escolar de un modo colaborativo, lo que implica construir culturas inclusivas con respeto a la diversidad. Por su parte, López (2006) señala que es necesario educar a la ciudadanía para la comprensión, respeto y promoción de las diferencias humanas para contribuir en el desarrollo de una cultura inclusiva. En un sentido pedagógico se deben efectuar estrategias para que el grupo aprenda a conocerse, 
valorarse, relacionarse consigo mismo, su entorno y con los demás, siendo base para una vida integral (Gamboa et al., 2020).

Lo señalado anteriormente permite plantear que las escuelas primarias en Hermosillo, Sonora, México, se encuentran ante el desafío de avanzar hacia una escuela inclusiva, porque se ubican en un proceso de ser escuelas integradoras, mas aún no se logra la participación efectiva de todos los miembros de la comunidad educativa. Razón por la cual es necesario un modelo de mejora de la escuela donde la planificación debe realizarse de modo colaborativo, comprendiendo la realidad y las perspectivas de todos los actores. De igual forma, la Secretaría de Educación Pública (2016), de acuerdo a los lineamientos de la reforma educativa, contempla entre uno de sus objetivos asegurar equidad y educación de calidad a todos los niños y niñas, al recomendar la reorganización de los centros escolares, recursos, procesos y acciones a seguir para promover e impulsar la educación inclusiva.

Se puede señalar que hubo limitantes de tipo temporal y espacial en la investigación debido a que se tenía contemplada una muestra mayor para el pilotaje; sin embargo, los horarios de los docentes no permitieron reunirlos a todos. Además, se pretendía acudir a otros municipios, pero por limitaciones en el tiempo de recolección de datos no se logró acudir a otras localidades de Sonora. Finalmente, se puede concluir que el modelo estructural en su conjunto predice un $36 \%$ de la educación inclusiva de los colectivos vulnerables, indicando una relación altamente significativa bidireccional entre la práctica y cultura inclusiva.

Dentro de los hallazgos encontrados se señala la demanda y necesidad de metodologías inclusivas que permitan ampliar los conocimientos y técnicas de trabajo con niños y niñas con algún tipo de vulnerabilidad; ello debido a que la gran gama de diversidad que se puede encontrar se genera un rechazo y estigma social. Lo anterior será un área de posible exploración para próximas investigaciones, incluyendo el estudio de las variables de actitudes, percepción y significado social, así como ampliar la muestra al incorporar tanto a los padres de familia y a los directivos como líderes educativos. En conclusión, la educación y la formación de los estudiantes es todavía un reto ante una sociedad cada vez más diversa y con desafíos para toda la comunidad educativa. 


\section{Agradecimientos}

Al Programa para el Desarrollo Profesional Docente, Tipo Superior del Gobierno de México por su apoyo y financiamiento para la realización de la investigación.

\section{Referencias}

Barrero, A. M. (2016). Lo visible e invisible de la diversidad en la educación infantil. Infancias Imágenes, 15(2), 262-270. https://doi.org/10.14483/udistrital.jour.infimg.2016.2.a07 Barriga, C., Rodríguez, C., \& Ferreira, R. (2019). Factors that bias teacher expectations: Findings from Chile. Revista Latinoamericana de Psicología, 51(3), 171-180. http:// dx.doi.org/10.14349/rlp.2019.v51.n3.4

Bentler, P. (2006). EQS 6 Structural Equations Program Manual. Multivariate Software. Blanco, R. (2015). Hacia una escuela para todos y con todos. Unesco.

Booth, T., \& Ainscow, M. (200o). Guía para la evaluación y mejora de la educación inclusiva: desarrollando el aprendizaje y la participación en las escuelas. Universidad Autónoma de Madrid.

Booth, T., \& Ainscow, M. (2011). Index para la inclusión: guía para la evaluación y mejora de la educación inclusiva. Consorcio Universitario para la Educación Inclusiva.

Campa, R., \& Contreras, C. (2018). Aspectos psicosociales asociados en el proceso de inclusión educativa durante la infancia intermedia. Infancias Imágenes, 17(1), 9-24. https://doi.org/10.14483/16579089.12535

Cara, M., Sola, T., Aznar, I., \& Fernández, F. (2015). Análisis organizativo de las clases de inclusión escolar en Francia. Revista Latinoamericana de Ciencias Sociales, Niñez y Juventud, 13(2), 795-807. https://doi.org/10.1160o/1692715x.13217200314

Casanova, M. (2017). Educación inclusiva en las aulas. La Muralla.

Consejo Nacional de Evaluación de la Política de Desarrollo Social. (2016). Pobreza en México. Autor.

Echeita, G. (2013). Inclusión y exclusión educativa. De nuevo «voz y quebranto». Reice. Revista Iberoamericana sobre Calidad, Eficacia y Cambio en Educación, 11(2), 99-118.

Echeita, G., \& Ainscow, M. (2011). La educación inclusiva como derecho: marco de referencia y pautas de acción para el desarrollo de una revolución pendiente. Tejuelo, Revista de Didáctica de la Lengua y la Literatura, (12), 26-46. 
Encuesta Nacional de Salud y Nutrición. (2016). Resultados por entidad federativa. Instituto Nacional de Salud Pública.

Euan, E., \& Echeverría, R. (2016). Evaluación psicopedagógica de menores con necesidades educativas especiales: una propuesta metodológica interdisciplinaria. Revista Latinoamericana de Ciencias Sociales, Niñez y Juventud, 14(2), 1103-1117.

Fjeld, A., \& Quintana, L. (2019). Reinstitucionalización, formas de vida y acciones igualitarias: reinvenciones de los común hoy contra el capitalismo neoliberal. Revista de Estudios Sociales, (70), 2-9. https://doi.org/10.7440/res70.2019.01

Gamboa, R., Bernal, M., Gómez, M., Gutiérrez, M., Monreal, C., \& Muñoz, V. (2020). Corporeidad, motricidad y propuestas pedagógico-prácticas en aulas de educación infantil. Revista Latinoamericana de Ciencias Sociales, Niñez y Juventud, 18(1), 1-22. http://dx.doi.org/10.1160o/1692715x.18101

González, M., \& Londoño, D. (2019). Estrategias pedagógicas de literacidad: experiencia significativa en una Institución Educativa de Boyacá. Revista Latinoamericana de Ciencias Sociales, Niñez y Juventud, 17(1), 253-268. https://dx.doi.org/ 10.11600/1692715X.17115

Guillén, M., \& Valenzuela, B. (2015). Diversidad e inclusión educativa en Sonora, México: reto en construcción. En B. Valenzuela, M. Guillén, \& A. Medina. Procesos educativos: desafíos y retos en siglo XXI. Pearson.

Hernández, R., Fernández, C., \& Baptista, P. (2010). Metodología de la investigación. McGraw-Hill.

Instituto Nacional de Estadística y Geografía. (2015). Principales resultados de la Encuesta Intercensal 2015, Sonora. Autor.

Jiménez, F., Lalueza, J., \& Fardella, C. (2017). Aprendizajes, inclusión y justicia social en entornos educativos multiculturales. Redie, Revista Electrónica de Investigación Educativa, 19(3), 10-23. https://doi.org/10.24320/redie.2017.19.3.830

Leithwood, K. (2009). ¿Cómo liderar nuestras escuelas? Aportes desde la investigación. Fundación Chile.

López, A. (2018). La escuela inclusiva: el derecho a la equidad y la excelencia educativa. Universidad del País Vasco.

López, M. (2006). La ética y la cultura de la diversidad en la escuela inclusiva. Sinéctica, $29,8-25$.

Marshesi, A., Blanco, R., \& Hernández, L. (coords.) (2014). Avances y desafíos de la educación inclusiva en Iberoamérica. Organización de Estados Iberoamericanos para la Educación, la Ciencia y la Cultura. 
Mateus, L., Vallejo, D., Obando, D., \& Fonseca, L. (2017). Percepción de las prácticas y de la cultura inclusiva en una comunidad escolar. Avances en Psicología Latinoamericana, 35(1), 177-191. http://dx.doi.org/10.12804/revistas.urosario.edu.co/apl/a.4854

Monje, C. (2011). Metodología de la investigación cuantitativa y cualitativa: guía didáctica. Universidad Sur colombiana.

Montiel, G., \& Arias, L. (2017). Práctica docente en atención de los alumnos con necesidades educativas especiales. Revista Electrónica sobre Tecnología, Educación y Sociedad, $4(7), 1-14$.

Ocampo, A. (2019). Educación inclusiva: una teoría sin disciplina. Legados y recuperación de saberes diaspóricos para una epistemología pluritópica. Revista Boletín Redipe, 8(9), 42-88. https://doi.org/10.36260/rbr.v8ig.814

Ordóñez, G. (2018). Discriminación, pobreza y vulnerabilidad: los entresijos de la desigualdad social en México. Región y Sociedad, 30(71), 1-30. https://doi.org/10.22198/ rys.2018.71.2377

Romero, S., García, I., Rubio, S., Martínez, A., \& Flores, V. (2018). Influencia del tipo de escuela (especial o regular) sobre el aprovechamiento académico del alumnado con necesidades educativas especiales. Universitas Psychologica, 17(1), 1-11. https://doi.org/ 10.11144/Javeriana.upsy17-1.itee

Secretaría de Educación Pública. (2016). El modelo educativo 2016: el planteamiento pedagógico de la reforma educativa. Autor.

Secretaría de Educación Pública. (2017). Aprendizajes clave para la educación integral. Autor.

Secretaría de Educación Pública. (2018). Sistema interactivo de consulta de estadística educativa. Autor.

Serrano, D., Ochoa, A., \& Arcos, E. (2019). Conceptualizaciones, perspectivas y referentes de la participación en la educación primaria, México. Revista Latinoamericana de Ciencias Sociales, Niñez y Juventud, 17(2), 1-22. https://doi.org/10.1160o/1692715x.17202

Subirats, J. (2013). Una visión desde las políticas públicas en pleno cambio de época y con riesgos significativos de exclusión social. Revista Internacional Animación, Territorios y Prácticas Socioculturales, (5), 13-26.

Valenzuela, B. A., Guillén, M., \& Campa, R. (2014). Recursos para la inclusión educativa en el contexto de educación primaria. Infancias Imágenes, 13(2), 64-75. https:// doi.org/10.14483/udistrital.jour.infimg.2014.2.206 